\title{
The Northern HIPASS Optical/IR Catalogue (NOIRCAT)
}

\author{
O. Ivy Wong ${ }^{1,2}$, Rachel L. Webster ${ }^{1}$, Meryl Waugh ${ }^{1}$, \\ Virginia A. Kilborn ${ }^{3}$ and Lister Staveley-Smith ${ }^{4}$ \\ ${ }^{1}$ School of Physics, University of Melbourne, VIC 3010, AUSTRALIA \\ email: iwong@ph.unimelb.edu.au \\ ${ }^{2}$ Australia Telescope National Facility, CSIRO PO Box 76, Epping, NSW 1710, AUSTRALIA \\ ${ }^{3}$ Swinburne University of Technology, PO Box 218, Hawthorn, VIC 3122, AUSTRALIA \\ ${ }^{4}$ School of Physics, University of Western Australia, 35 Stirling Hwy, Crawley, WA 6009, \\ AUSTRALIA
}

\begin{abstract}
We present the Northern HIPASS Optical/Infrared Catalogue (NOIRCAT), an optical/near-infrared counterpart catalogue for the Northern HIPASS catalogue (NHICAT). Of the 1002 sources in NHICAT, 655 (66\%) have optical counterparts with matching optical velocities. A further 87 sources have optical counterparts with matching velocities from previous radio emission line surveys. Assuming a dark galaxy to be an isolated Hi source with no detectable stars, no candidate dark galaxies have been confirmed.
\end{abstract}

Keywords. observational, catalogues, surveys, radio lines, galaxies.

\section{Northern HIPASS}

The Hi Parkes All-Sky Survey (HIPASS) survey is the largest blind Hi survey, covering $71 \%$ of the total sky using the 21-cm multibeam receiver (Staveley-Smith et al. 1996) on the Parkes Radio Telescopet. Northern HIPASS surveys the entire sky within the declination range $+2^{\circ}<\delta<+25.5^{\circ}$ whereas Southern HIPASS covers the entire Southern sky below a declination of $+2^{\circ}$ (Meyer et al. 2004). The Northern HIPASS catalogue (NHICAT; Wong et al. 2006) detected 1002 galaxies based solely on the Hi content. The observation and reduction methods are exactly the same as Southern HIPASS and can be found in detail in Barnes et al. (2001). NHICAT has been found to be $95 \%$ complete at peak flux $95 \mathrm{mJy}$ and at an integrated flux $15 \mathrm{Jy} \mathrm{km} \mathrm{s}^{-1}$ (NHICAT; Wong et al. 2006). The reliability of this catalogue at a $95 \%$ level is at peak flux 36 mJy (NHICAT; Wong et al. 2006).

Here, we present the Northern HIPASS optical and near-IR catalogue (NOIRCAT) a catalogue of optical and near-infrared counterparts to the HI galaxies in NHICAT. NOIRCAT is analogous to the HIPASS Optical Catalogue (HOPCAT; Doyle et al. 2005) which is a catalogue of optical counterparts for HICAT.

\section{NOIRCAT}

The large Southern sky surveys used to generate HOPCAT, such as 6dF and SUPERCOSMOS, are not available for constructing NOIRCAT. Northern analogues of $6 \mathrm{dF}$ and SUPERCOSMOS do not exist-most large, recent optical surveys such as SDSS do not cover the entire Northern sky. Hence, NED is used as our source catalogue for optically

$\dagger$ The Parkes Telescope is part of the Australia Telescope which is funded by the Commonwealth of Australia for operation as a National Facility managed by CSIRO. 
Table 1. Description and tally of the NOIRCAT match categories (flags).

\begin{tabular}{clc}
\hline Flag & Description & Number \\
\hline 1 & Single optical velocity match with 2MASS counterpart & 414 \\
2 & Single optical velocity match without 2MASS counterpart & 126 \\
3 & Multiple optical velocity matches where all matches have 2MASS counterparts & 63 \\
4 & Multiple optical velocity matches where 1 or more matches are without 2MASS counterpart(s) & 52 \\
$5 \mathrm{a}$ & Match with higher angular resolution Hi velocity and optically visible galaxy & 87 \\
$5 \mathrm{~b}$ & Only positional matches available to NED galaxies & 219 \\
$5 \mathrm{c}$ & Neither velocity nor positional matches to any galaxies listed in NED & 41 \\
\hline
\end{tabular}

matching the Northern HIPASS detections. It should be noted that we used the October 2006 version of NED. To improve optical detection limits, we also used the 2MASS near-infrared catalogue.

For NOIRCAT, the primary method for determining the optical/near-infrared matches was by "interactive" cataloguing, after an automated search of the NED and 2MASS catalogues. The four criteria used for determining a match during the interactive process are:

(a) Optical sources must be within $7.5^{\prime}$ of the HIPASS centre.

(b) Where there are multiple source names referring to the same source (e.g. SDSS and APM nomenclatures), the non-SDSS/APM reference is preferred.

(c) Optical velocity matches are made when the optical velocity (including errors) and the HIPASS heliocentric velocity (including errors) agreed to within $100 \mathrm{~km} \mathrm{~s}^{-1}$.

(d) For optical velocities without published errors in NED, a match is recorded when the published optical velocity is within $150 \mathrm{~km} \mathrm{~s}^{-1}$ of the HIPASS velocity profile.

Table 1 describes the match categories into which the resulting matches are classified. A tally of the NHICAT sources in each match category is also provided.

\section{Summary}

A thorough description and analysis of NOIRCAT can be found in Wong et al. 2007. This catalogue will be submitted to the NASA/IPAC Extragalactic Database and will also be made publicly available online at http://hipass . aus-vo.org.

\section{Acknowledgements}

O. I. W. acknowledges the financial support from the ATNF (CSIRO), the IAU and the Astronomical Society of Australia. This research has made use of the NASA/IPAC Extragalactic Database (NED) which is operated by the Jet Propulsion Laboratory, California Institute of Technology, under contract with the National Aeronautics and Space Administration. This research has also made use of data products from the Two Micron All Sky Survey, which is a joint project of the University of Massachusetts and the Infrared Processing and Analysis Center/California Institute of Technology, funded by the National Aeronautics and Space Administration and the National Science Foundation.

\section{References}

Barnes, D. G. et al. 2001, MNRAS 322, 486

Doyle, M. T. et al. 2005, MNRAS 361, 34

Meyer, M. J. et al. 2004, MNRAS 350, 1195

Staveley-Smith, L. et al. 1996, PASA 13, 243

Wong, O. I. et al. 2006, MNRAS 371, 1855

Wong, O. I., Webster, R. L., Kilborn, V. A., Waugh, M. \& Staveley-Smith, L. 2007, in preparation 